\title{
Comparative Analysis of non-coding smallRNAs in Pseudomonas aeruginosa Keratitis Strains with Different Antibiotic Susceptibility
}

\author{
Kathirvel Kandasamy ${ }^{1,3}$, Prajna Lalitha ${ }^{2}$, Bharanidharan Devarajan ${ }^{\text {* }}$ \\ ${ }^{1}$ Department of Microbiology and Bioinformatics, Aravind Medical Research Foundation, \\ Madurai, India. \\ ${ }^{2}$ Department of Microbiology, Aravind Eye Hospital, Madurai, India. \\ ${ }^{3}$ School of Chemical and Biotechnology, SASTRA deemed to be university, Thanjavur, India.
}

*Correspondence:

Bharanidharan Devarajan

Scientist, Department of Microbiology and Bioinformatics

Aravind Medical Research Foundation, 1, Anna Nagar,

Madurai, Tamil Nadu, India - 625020

Phone: 0452-4356550

Email: bharani@aravind.org; bharanid@gmail.com 


\section{Introduction}

Pseudomonas aeruginosa, is a ubiquitous gram-negative bacterium which belongs to Pseudomonadaceae family that causes opportunistic or nosocomial infections in immunocompromised individuals [1,2]. P. aeruginosa can transform to infectious human pathogen from being an environmental isolate such as water and soil [1]. While its ability to causing chronic pulmonary inflammation in cystic fibrosis patients, urinary and respiratory tract infections is well studied, whereas $P$. aeruginosa in bacterial keratitis is relatively understudied. Human corneal infections by $P$. aeruginosa are majorly related to improper contact lens wear and handling, and other risk factors for keratitis in non-contact lens wearers includes ocular surgery, ocular trauma and earlier ocular surface disease [3-6]. Treating the infection of $P$. aeruginosa is tough due to its resistance to multiple class of antibiotics, which resulted from complex transcriptional regulatory networks it possesses and expressing different sets of genes in different environments to facilitate adaption and growth in antibiotic induced stressful environments $[7,8]$. In recent years, increased multi-drug resistance (MDR) or extensively drug resistance (XDR) of $P$. aeruginosa keratitis strains has been reported by several studies [9-13]. However, the emerging MDR/XDR $P$. aeruginosa keratitis strains were resistant to aminoglycosides, carbapenems and quinolones, the options for its treatment are very limited.

Bacterial non-coding smallRNAs (sRNAs) are between 50 and 300 nt long in length, induces post transcriptional events and an increased number of sRNAs has been reported in various pathogenic bacteria including Staphylococcus aereus, Escherichia coli and P. aeruginosa from the past decade [14-17]. Similar to eukaryotic miRNAs, bacterial sRNAs have multiple targets and regulate them in trans as well as cis acts by base-pairing in anti-sense orientation with complementary sequence of its target mRNAs [18]. Bacterial sRNA commonly required the smlike RNA-binding protein called Hfq, which interacts with both sRNA and mRNA and facilitates the interaction between anti-sense sRNA and their binding target mRNAs in post transcriptionally [19,20]. Hfq also can serve alone as translational repressor of mRNA [21,22]. Several sRNAs, has been identified to regulate various physiological processes in $P$. aeruginosa, including biofilm formation, quorum sensing [17,21]. However, the regulatory mechanism of this non-coding sRNAs in MDR/XDR pathways of $P$. aeruginosa keratitis strains is not yet studied. 
The aim of the present study was to identify the bacterial sRNAs in available $P$. aeruginosa keratitis genomes and study their regulatory functions in MDR/XDR pathways.

\section{Methods}

\subsection{Bacterial strains and antibiotic susceptibility profile}

In total five $P$. aeruginosa keratitis genomes from our previous study [9] and 41 reported complete genomes of $P$. aeruginosa keratitis strains from India, Australia and England [10-13] were used for non-coding sRNA identification in the present study. All genomes were retrieved in GenBank format and dated before October 2020. The details of antibiotic susceptibility of each strain used in the present study was obtained from respected reports, if available. Based on the susceptibility towards number of antimicrobial agents, the strains were defined as non-MDR, MDR and XDR [23]. Briefly, the strains which non-susceptible to $\geq 1$ agent in $\geq 3$ antimicrobial categories were MDR and the strains which non-susceptible to $\geq 1$ agent in all but $\leq 2$ antimicrobial categories were defined as XDR. The complete details of $P$. aeruginosa keratitis genomes used in this study are shown in Table 1.

\subsection{Phylogeny and sRNA identification}

A core genome based maximum-likelihood tree of $46 P$. aeruginosa keratitis genomes was created using Parsnp version 1.2 in Harvest Suite [24]. The P. aeruginosa complete genomes PAO1 [1], UCBPP-PA14 [25] and taxonomic outliner PA7 [26] also included in phylogeny. Phylogenetic tree visualization and figure generation was done using the iTOL software [27]. Non-coding smallRNAs in all $46 P$. aeruginosa keratitis genomes were identified by aligning each draft genome against Bacterial smallRNA Database (BSRD) using BLAST Ring Image Generator (BRIG) with $90 \%$ maximum and $80 \%$ minimum sequence similarity for the best hit [28]. Totally 130 known sRNAs from $P$. aeruginosa reference genome PAO1 and virulence genome UCBPP-PA14 were manually curated from BSRD for sRNA identification.

\subsection{Target prediction and functional analysis}

Bacterial binding target genes of identified sRNAs were predicted using various target prediction servers such as TargetRNA2 [29] and IntaRNA [30]. Genes with high affinity of binding with sRNAs selected for the pathway analysis using DAVID [31]and KEGG [32] databases. Gene 
ontology (GO) terms of identified sRNAs in Biological Process (BP), Cellular Component (CC) and Molecular Function (MF) were predicted by their binding target genes using DAVID.

\section{Results and Discussion}

\subsection{Antibiotic susceptibility profile and genome characteristics}

Totally, eighteen, nineteen and eight P. aeruginosa strains used in the present study were found as non-MDR, MDR and XDR respectively (Table 1). Strains with intermediate resistance to antimicrobial agents also categorized as resistant for subsequent analysis. Eight (30\%) out of Twenty-six and Ten (52\%) out of Nineteen P. aeruginosa strains from India and Australia were identified as not-MDR. Whereas, 10 (38\%) Indian and 9 (47\%) Australian strains were identified as MDR showing resistant to at least more than one antibiotic from $\geq 3$ antimicrobial categories. Eight Indian strains were identified as XDR showing resistant to at least more than one antibiotic from all but $\leq 2$ tested antimicrobial categories. Overall, Indian P. aeruginosa strains are more resistant to antibiotics compared to others. Among all tested antibiotics to keratitis P. aeruginosa strains, ceftazidime resistance in 25, ciprofloxacin resistance in 29 and imipenem resistance in 30 strains were observed (Figure 1). Strain PA193 from India showed no resistance to any tested antibiotics. All strains were sensitive to colistin. Out of 46 strains, 23 (50\%) carried ExoU, 21(45\%) carried ExoS and two (5\%) strains carried both ExoU and ExoS, exotoxins for their virulence. Indian strain with poor clinical outcome BK5, reported to have non-synonyms mutation in ExoU protein which leads loss of ExoU gene function. Australian strain PA126 harbored both ExoU and ExoS, while no mutations were found in ExoU gene. Two not-MDR Australian strains PA126 and PA169 found to be carried ExoU. Whereas, Eight MDR/XDR strains from Australia (6) and India (2) were found to be carried ExoS (Table 1). These findings indicate ExoS carrying strains might have additional strain-specific genes for their virulence mechanism. Out of 46 strains used in this study, the clinical outcome of five strains were poor and remaining strain's clinical outcomes were either good (healed) or not available. 


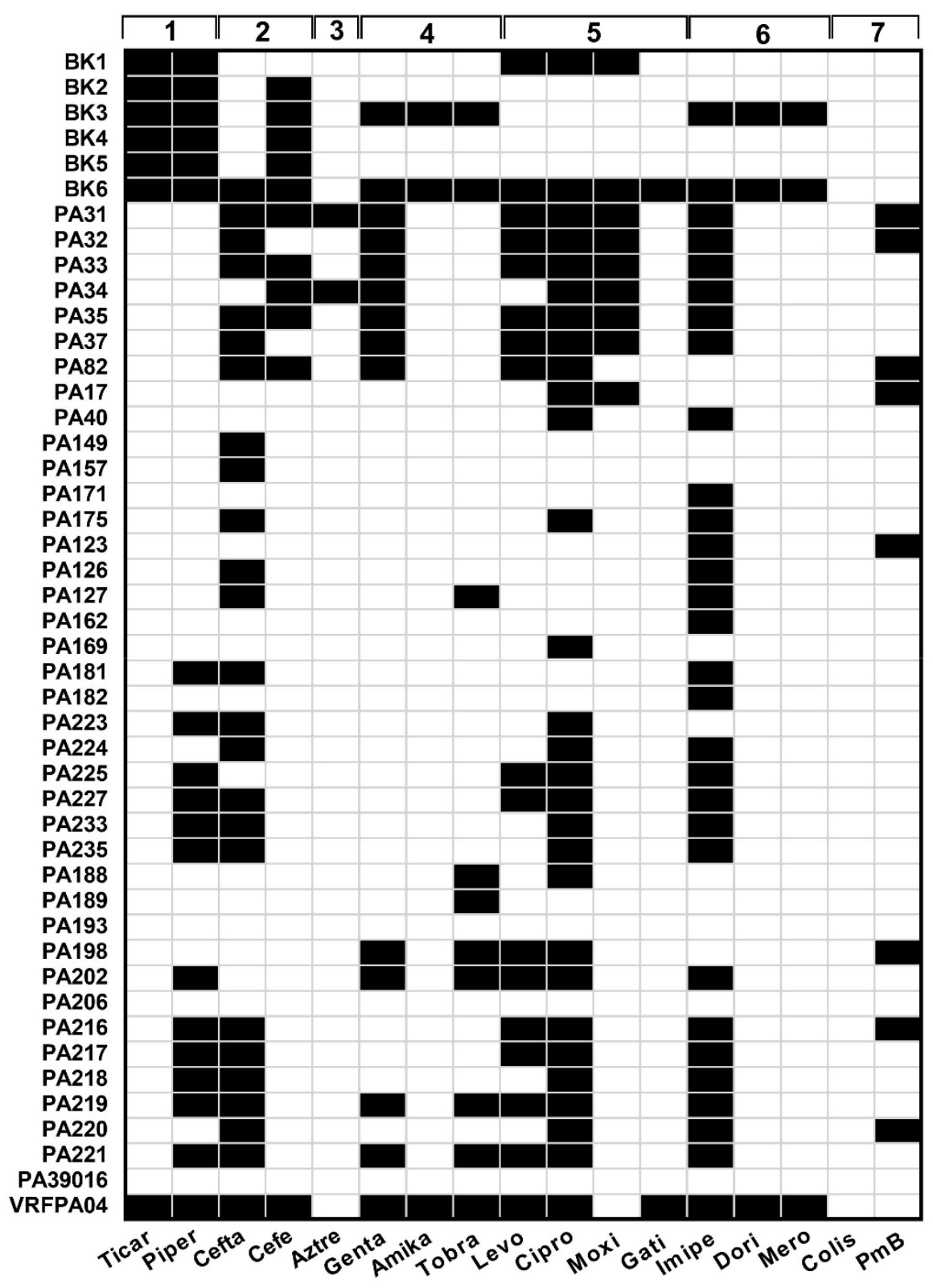

Figure 1. The antibiotic susceptibility profile of $P$. aeruginosa keratitis strains used in this study. Class of antimicrobial agents, [1] Penicillins/B-lactamase inhibitors; [2] Cephalosporins; [33] Monobactams; [4] Aminoglycosides; [5] fluoroquinolones; [6] Carbapenems; [7] Polymyxins.

The genome size of keratitis $P$. aeruginosa strains ranged from $6.2 \mathrm{Mb}$ to $8.3 \mathrm{Mb}$. The minimum and maximum number of contigs were 1 to 1917 and the average number of protein 
coding genes were 6285 from all $P$. aeruginosa strains used in this study (Table 1). The percentage of GC content of all strains were 66 as expected.

Table 1. The complete genomic features, clinical outcome and regions of P. aeruginosa keratitis strains used in this study.

\begin{tabular}{|c|c|c|c|c|c|c|c|c|c|}
\hline Strain ID & $\begin{array}{l}\text { Genome } \\
\text { size }(\mathrm{Mb})\end{array}$ & $\begin{array}{l}\text { No. of } \\
\text { Contigs }\end{array}$ & CDs & $\begin{array}{l}\text { GC } \\
\text { (\%) }\end{array}$ & Type & UST & $\begin{array}{l}\text { Clinical } \\
\text { Outcome }\end{array}$ & Region & $\begin{array}{l}\text { GenBank } \\
\text { accession }\end{array}$ \\
\hline BK1 & 6.4 & 163 & 6736 & 66 & Not-MDR & ST & Poor & India & JBTQ00000000.1 \\
\hline $\mathrm{BK} 2$ & 6.3 & 79 & 6037 & 66 & Not-MDR & ST & Good & India & GCA_002243265.1 \\
\hline BK3 & 7.1 & 161 & 7009 & 66 & MDR & UT & Poor & India & GCA_002242775.1 \\
\hline BK4 & 6.5 & 248 & 6141 & 66 & Not-MDR & ST & Poor & India & GCA_002242885.1 \\
\hline BK5 & 6.3 & 133 & 6055 & 66 & Not-MDR & UST & Poor & India & GCA_002242915.1 \\
\hline BK6 & 7.1 & 202 & 6722 & 66 & XDR & UT & Good & India & GCA_002242855.1 \\
\hline PA31 & 7.1 & 137 & 6619 & 66 & XDR & UT & NA & India & GCA_003332785.1 \\
\hline PA32 & 7.1 & 155 & 6611 & 66 & XDR & UT & NA & India & GCA_003332735.1 \\
\hline PA33 & 7.1 & 166 & 6609 & 66 & MDR & UT & NA & India & GCA_003332715.1 \\
\hline PA34 & 6.8 & 130 & 6326 & 66 & XDR & UT & NA & India & GCA_003332705.2 \\
\hline PA35 & 7.1 & 156 & 6611 & 66 & MDR & UT & NA & India & GCA_003332755.1 \\
\hline PA37 & 7.1 & 241 & 6645 & 66 & MDR & UT & NA & India & GCA_003332665.1 \\
\hline PA82 & 6.3 & 64 & 5810 & 66 & MDR & UT & NA & India & GCA_003332645.1 \\
\hline PA17 & 6.3 & 60 & 5825 & 66 & Not-MDR & ST & NA & Australia & GCA_003332795.1 \\
\hline PA40 & 6.2 & 109 & 5700 & 66 & Not-MDR & ST & NA & Australia & GCA_003332655.1 \\
\hline PA149 & 6.3 & 59 & 5745 & 66 & Not-MDR & ST & NA & Australia & GCA_003332625.1 \\
\hline PA157 & 6.2 & 56 & 5708 & 66 & Not-MDR & ST & NA & Australia & GCA_003332575.1 \\
\hline PA171 & 6.3 & 60 & 5812 & 66 & Not-MDR & ST & NA & Australia & GCA_003332565.1 \\
\hline PA175 & 6.7 & 62 & 6181 & 66 & MDR & UT & NA & Australia & GCA_003332455.1 \\
\hline PA123 & 6.3 & 86 & 5858 & 66 & Not-MDR & UT & NA & Australia & GCA_009727505.1 \\
\hline PA126 & 6.4 & 81 & 6007 & 66 & Not-MDR & UST & NA & Australia & GCA_009727515.1 \\
\hline PA127 & 6.3 & 86 & 5858 & 66 & MDR & UT & NA & Australia & GCA_009727535.1 \\
\hline PA162 & 6.6 & 87 & 6131 & 66 & Not-MDR & UT & NA & Australia & GCA_009727485.1 \\
\hline PA169 & 6.3 & 50 & 5918 & 66 & Not-MDR & UT & NA & Australia & GCA_009727465.1 \\
\hline PA181 & 7.1 & 122 & 6619 & 66 & MDR & ST & NA & Australia & GCA_009727425.1 \\
\hline PA182 & 6.8 & 58 & 6392 & 66 & Not-MDR & ST & NA & Australia & GCA_009727385.1 \\
\hline PA223 & 6.9 & 108 & 6408 & 66 & MDR & ST & NA & Australia & GCA_014673185.1 \\
\hline PA224 & 6.4 & 151 & 5811 & 66 & MDR & ST & NA & Australia & GCA_014673235.1 \\
\hline PA225 & 7.2 & 294 & 6607 & 66 & MDR & ST & NA & Australia & GCA_014672955.1 \\
\hline PA227 & 7.1 & 102 & 6529 & 66 & MDR & ST & NA & Australia & GCA_014673145.1 \\
\hline PA233 & 6.3 & 92 & 5745 & 66 & MDR & UT & NA & Australia & GCA_014673245.1 \\
\hline PA235 & 6.2 & 56 & 5719 & 66 & MDR & ST & NA & Australia & GCA_014672935.1 \\
\hline
\end{tabular}




\begin{tabular}{|l|l|l|l|l|l|l|l|l|l|}
\hline PA188 & 6.3 & 56 & 5818 & 66 & Not-MDR & ST & NA & India & GCA_009727395.1 \\
\hline PA189 & 6.3 & 59 & 5820 & 66 & Not-MDR & ST & NA & India & GCA_009727345.1 \\
\hline PA193 & 6.3 & 66 & 5888 & 66 & Not-MDR & ST & NA & India & GCA_009727335.1 \\
\hline PA198 & 7.1 & 119 & 6727 & 66 & MDR & UT & NA & India & GCA_009727325.1 \\
\hline PA202 & 7.1 & 368 & 6883 & 66 & MDR & UT & NA & India & GCA_009727285.1 \\
\hline PA206 & 6.5 & 55 & 6047 & 66 & Not-MDR & ST & NA & India & GCA_009727245.1 \\
\hline PA216 & 8.3 & 1917 & 8943 & 66 & XDR & ST & NA & India & GCA_009727295.1 \\
\hline PA217 & 6.8 & 132 & 6482 & 66 & MDR & UT & NA & India & GCA_009727225.1 \\
\hline PA218 & 6.3 & 77 & 5840 & 66 & MDR & ST & NA & India & GCA_009727235.1 \\
\hline PA219 & 7.4 & 166 & 7122 & 66 & XDR & UT & NA & India & GCA_009727125.1 \\
\hline PA220 & 6.6 & 90 & 6144 & 66 & MDR & UT & NA & India & GCA_009727165.1 \\
\hline PA221 & 7.2 & 294 & 6829 & 66 & XDR & UT & NA & India & GCA_009727135.1 \\
\hline PA39016 & 6.8 & 486 & NA & NA & NT & UT & NA & England & AEEX00000000.1 \\
\hline VRFPA04 & 6.8 & 1 & 5778 & NA & XDR & UT & Poor & India & GCA_000473745.1 \\
\hline
\end{tabular}

\subsection{Phylogeny}

Core genome based phylogenetic tree showed that all 46 P. aeruginosa keratitis strains were clustered into two groups, where PA7 and PA206 as outliner as expected (Figure 2). These phylogenetic results are consisted and similar with previous studies which have also shown that $P$. aeruginosa strains from different sources tend to cluster into two groups [10,34-36]. All MDR and XDR strains were clustered in group 1, along with two not-MDR strains PA162 and PA169. Group 2 contains 25 strains, where 16 and 9 were not-MDR and MDR/ XDR strains, respectively. Surprisingly, the strains were not grouped based on either antibiotic susceptibility profile or T3SS exotoxin. Group 1 tend to be smaller than group 2 and contains a greater number of Indian P. aeruginosa keratitis strains. 


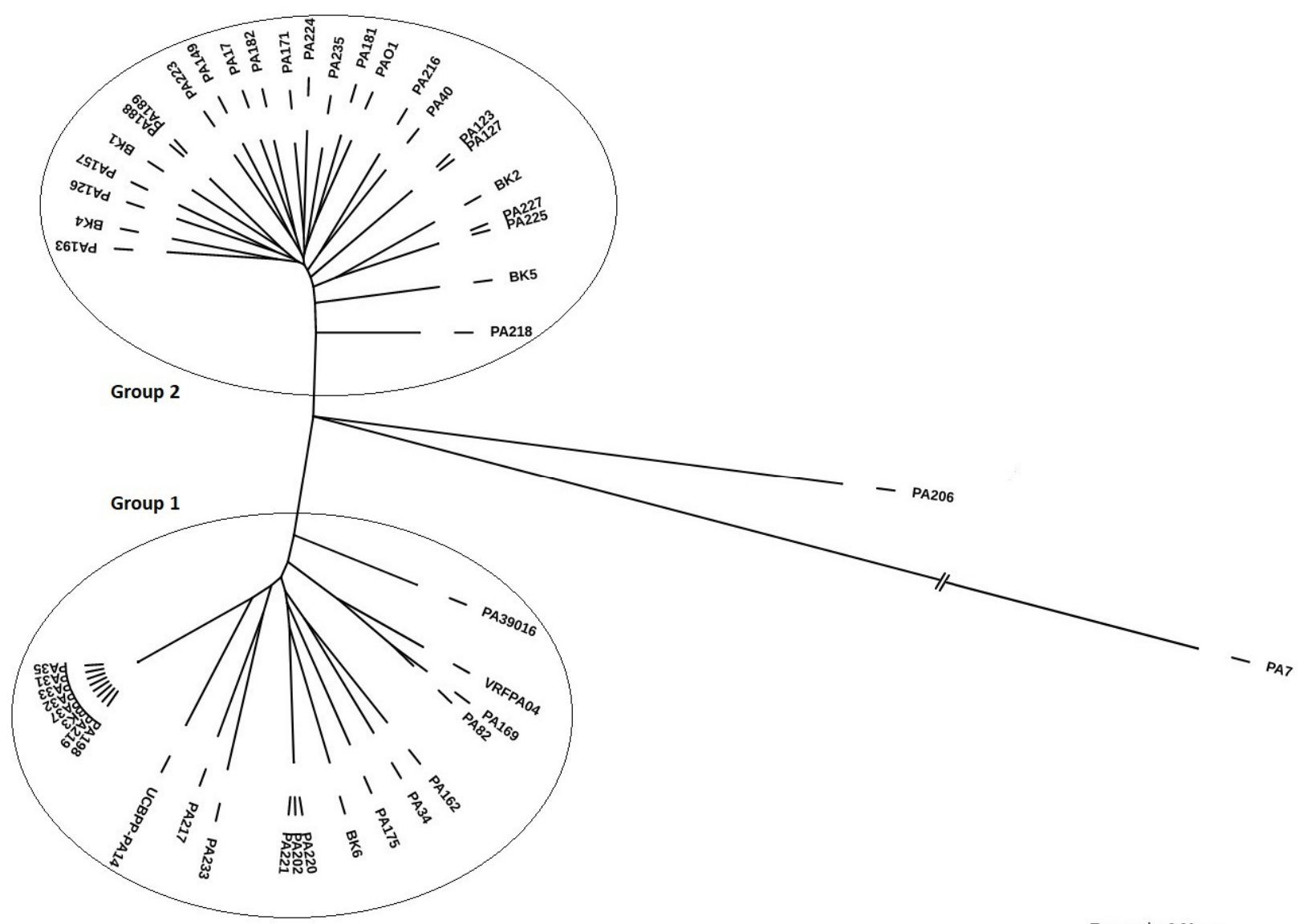

Tree scale: $0.01 \mapsto$

Figure 2. A core genome-based maximum-likelihood phylogenetic tree constructed by parsnp.

\subsection{Bacterial non-coding sRNAs and their binding targets}

We identified common and strain-specific non-coding sRNAs in P. aeruginosa keratitis genomes by aligning with 130 known bacterial sRNAs from PAO1 and UCBPP-PA14 reference trains using BRIG tool as shown in Figure 3. SPA0017 from UCBPP-PA14 virulent strain, was not detected or partial sequence was identified in all keratitis strains used in this study. sRNAs, SPA0010 and SPA0018 was detected only in PA39016, VRFPAO4 and UCBPP-PA14 virulent strains. The complete sequence of SPA0012 from UCBPP-PA14, was identified only in PA223 and VRFPA04. Complete sequence of UCBPP-PA14 virulent strain sRNA, SPA0011 was detected in PA175 and partially in PA223, PA225 and PA227 strains. The sRNAs, SPA0013 and SPA0019 from UCBPP-PA14 strain was not detected in any keratitis strains of the present study. Whereas, SPA0014 was detected with complete sequence identity in BK4, PA149, PA224, 
PA225, PA227 and VRFPAO4 strain. The sRNA, SPA0021 was identified in 6 Not-MDR and 18 MDR/XDR strains along with UCBPP-PA14. Several well-known P. aeruginosa sRNAs (PhrX, PhrY, PrrB, PrrH, PrrF, PrrF2 and CrcZ), reported to have role in pathogenicity was detected in all keratitis strains of the present study along with PAO1 and UCBPP-PA14 reference strains. Complete absence of PhrD was observed in all keratitis strains including UCBPP-PA14.
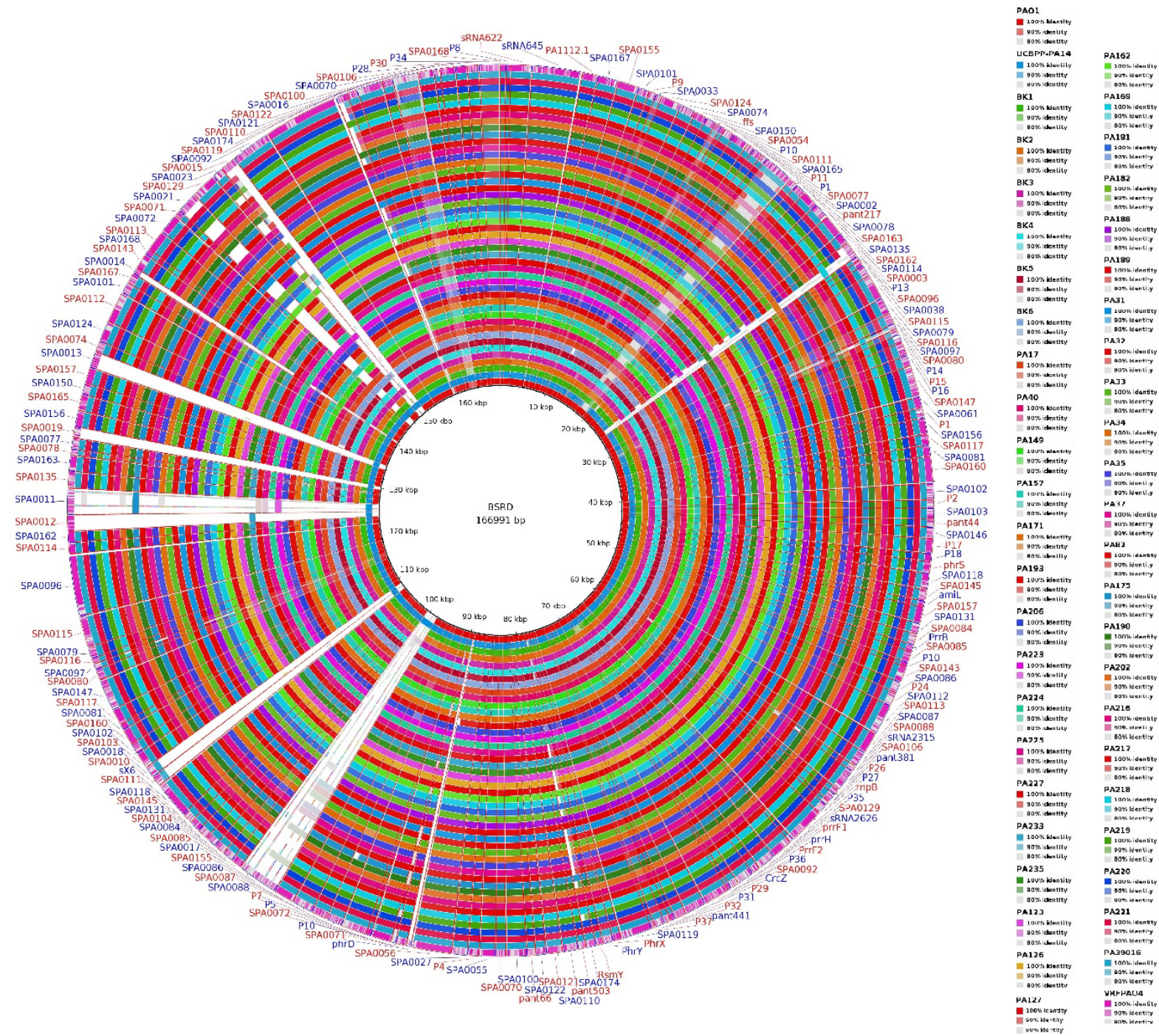

Figure 3. A BLAST ring circular representation of bacterial sRNAs from all $P$. aeruginosa keratitis strains used in this study.

Interestingly, sRNA SPA0023 was found in majority of the MDR/XDR keratitis strains (BK3, BK6, PA31, PA32, PA33, PA34, PA35, PA37, PA198, PA202, PA216, PA217, PA219, PA220, PA221, PA223, PA39016 and VRFPAO4) along with not-MDR strains PA162, PA182. The 
sRNA SPA0023, fall within the pathogenicity island PAPI-1, which is cis-encoded antisense to RL033 (PA14_59840) gene, encoding hypothetical protein. A mutation in PA14_59840 has been reported with attenuated virulence [37]. Interestingly, majority of the imipenem resistant $P$. aeruginosa keratitis strains from the present study was found to be carried SPA0023 sRNA (18 out of 30 strains). The outer membrane porin protein OprD, identified as binding target of SPA0023 with high affinity of interaction. Negative regulation or inactivation of OprD, reported in increased imipenem resistance in P. aeruginosa [38-41]. Mutation analysis revealed that SPA0023 carrying $P$. aeruginosa keratitis strains contains a lesser number of amino acid changes in OprD protein than other strains. These findings indicate, imipenem resistance in SPA0023 carried strains might arose from the negative regulation or inhibition of OprD by SPA0023. However, functional studies are warranted with large number of $P$. aeruginosa keratitis strains to confirm the negative regulation of OprD by SPA0023 and imipenem resistance. The secondary minimum free energy (MFE) structure of SPA0023 was predicted using RNAfold server and shown in Supplementary Figure S1. The binding target genes of identified sRNAs were predicted by sequence-based target prediction tools TargetRNA2 and IntaRNA. Targets predicted by both tools were considered for pathway analysis and listed in Supplementary Table S1.

\subsection{Pathway analysis}

The pathways associated with binding target genes of common and uniquely shared sRNAs in keratitis $P$. aeruginosa strains were predicted using DAVID and KEGG pathway tools. Totally, 8 pathways were enriched significantly, with more than three target genes of sRNAs and listed in Table 2. The functions of predicted targets genes of sRNAs were identified in GO terms using DAVID and listed in Supplementary Table S2.

Table 2. Pathways associated with target genes of identified sRNAs in this study.

\begin{tabular}{|l|l|l|l|}
\hline Pathway name & Count & PValue & Genes \\
\hline Metabolic & 180 & $9.76 E-04$ & PYKF, APT, DGKA, GLK, GLTX, PCHA, LIPB, \\
pathways & & PGPA, DNAE, ACCC, CCON1, ACCD, ACCA, \\
& & ACCB, CMK, ACEA, GMK, SERC, ANSA, \\
& & GLCD, SERA, GLYA3, SDHC, FOLM, METE, \\
& & DAPE, HISA, DAPD, FUMC2, ILVE, ILVC, \\
& & ILVD, PGK, HISI, METX, METY, METZ, \\
& & PNTAB, BIOF, GCDH, WAAA, PAND, PANC, \\
& & KDSA, WAAC, WAAF, ACNB, COXB, ACNA, \\
\hline
\end{tabular}




\begin{tabular}{|c|c|c|c|}
\hline & & & $\begin{array}{l}\text { LPXC, UBIG, PANE, UBIE, MOAA1, CYSM, } \\
\text { MTLZ, WAAP, CYSK, MQOA, CATB, MQOB, } \\
\text { GCVP1, NDK, CATA, LPXK, GCVP2, NADC, } \\
\text { WAAG, HISC1, ACEE, HUTI, FABG, ARUB, } \\
\text { ARUC, MOAE, NRDA, XYLL, GLMS, ACOB, } \\
\text { SOXG, PCAC, RFAD, FABZ, HEME, GGT, } \\
\text { ATPF, HEMH, PCAG, HEML, RFAE, TAL, } \\
\text { PNCB1, PCAH, HEMN, GLPK, KYNA, PEPA, } \\
\text { HISF1, SPDH, PHNB, IDH, THRH, PHNA, } \\
\text { FOLE2, RPOZ, RIBB, PEPN, SPUI, SPED, } \\
\text { HOLA, ARUE, RIBD, PSD, GLGB, BDHA, } \\
\text { COBP, THID, THIC, COBH, BKDA1, GLGP, } \\
\text { COBD, COBB, THII, GDHB, RPOA, RPOB, } \\
\text { GDHA, THIE, PHZC2, LYSA, PHZC1, NUOL, } \\
\text { NUOM, HISH2, NUOI, NUOJ, NUOK, TRPA, } \\
\text { DADX, XDHB, PDXY, GCVT2, ARGE, PURD, } \\
\text { TRPF, TRPE, FAHA, ARGA, TRPC, PONA, } \\
\text { AROE, PYRH, AROC, PYRB, UREB, PURE, } \\
\text { FOLB, PURH, PYRD, PYRC, CCOQ1, LEUC, } \\
\text { PHNX, YGBB, LIUC, GATB, FABH2, LIUA, } \\
\text { PHOA, LIUD, RPIA, LDCA, YGBP, EPD, NUOD, } \\
\text { MURB, MURC, NUOA, NUOB }\end{array}$ \\
\hline $\begin{array}{l}\text { Biosynthesis of } \\
\text { secondary } \\
\text { metabolites }\end{array}$ & 79 & 0.046023 & $\begin{array}{l}\text { PYKF, HISF1, DGKA, GLK, GLTX, PCHA, } \\
\text { PHNB, IDH, THRH, PHNA, RIBB, ACCC, } \\
\text { ACCD, ACCA, ACCB, RIBD, ACEA, PSD, } \\
\text { GLGB, ANSA, GLCD, GLYA3, SDHC, METE, } \\
\text { BKDA1, HISA, GLGP, FUMC2, ILVE, ILVC, } \\
\text { ILVD, PGK, HISI, PHZC2, LYSA, PHZC1, ISPB, } \\
\text { PAND, PANC, HISH2, TRPA, ACNB, ACNA, } \\
\text { UBIG, PANE, UBIE, HCNA, GCVT2, CYSK, } \\
\text { ARGE, PURD, MQOA, GCVP1, MQOB, TRPF, } \\
\text { NDK, TRPE, GCVP2, ARGA, TRPC, HISC1, } \\
\text { AROE, AROC, ACEE, PURE, PURH, ARUC, } \\
\text { LEUC, KATE, YGBB, ACOB, RPIA, WRBA, } \\
\text { HEME, YGBP, HEMH, HEML, TAL, HEMN }\end{array}$ \\
\hline $\begin{array}{l}\text { Biosynthesis of } \\
\text { amino acids }\end{array}$ & 40 & 0.024351 & $\begin{array}{l}\text { PYKF, HISF1, HISH2, TRPA, ACNB, PHNB, } \\
\text { ACNA, IDH, THRH, PHNA, CYSM, ARGE, } \\
\text { CYSK, TRPF, TRPE, ARGA, TRPC, SPU, } \\
\text { HISC1, AROE, AROC, ARUC, LEUC, SERC, } \\
\text { SERA, GLYA3, METE, DAPE, HISA, DAPD, } \\
\text { ILVE, RPIA, ILVC, ILVD, PGK, HISI, PHZC2, } \\
\text { LYSA, PHZC1, TAL }\end{array}$ \\
\hline $\begin{array}{l}\text { Phenylalanine, } \\
\text { tyrosine and } \\
\text { tryptophan } \\
\text { biosynthesis }\end{array}$ & 11 & 0.054421 & $\begin{array}{l}\text { TRPF, TRPE, TRPC, PHZC2, TRPA, HISC1, } \\
\text { AROE, PHZC1, PHNB, AROC, PHNA }\end{array}$ \\
\hline
\end{tabular}




\begin{tabular}{|l|l|l|l|}
\hline $\begin{array}{l}\text { Lipopolysaccharide } \\
\text { biosynthesis }\end{array}$ & 10 & 0.017695 & $\begin{array}{l}\text { WAAP, RFAD, WAAA, WAAC, KDSA, LPXK, } \\
\text { WAAF, WAAG, LPXC, RFAE }\end{array}$ \\
\hline Mismatch repair & 8 & 0.034248 & $\begin{array}{l}\text { XSEB, MUTS, MUTL, UVRD, LIG, RECJ, } \\
\text { HOLA, DNAE }\end{array}$ \\
\hline $\begin{array}{l}\text { Streptomycin } \\
\text { biosynthesis }\end{array}$ & 5 & 0.053206 & RMLC, RMLD, RMLA, GLK, RMLB \\
\hline $\begin{array}{l}\text { Polyketide sugar } \\
\text { unit biosynthesis }\end{array}$ & 4 & 0.055425 & RMLC, RMLD, RMLA, RMLB \\
\hline
\end{tabular}

\section{Conclusion}

This study identified and compared the bacterial non-coding sRNAs in P. aeruginosa keratitis strains with different antibiotic susceptibility profile for the first time. Totally, 46 P. aeruginosa keratitis strains from different geographical regions were included for the sRNA identification and investigating their regulatory role in MDR/XDR pathways. Several well-studied $P$. aeruginosa sRNAs (PhrX, PhrY, PrrB, PrrH, PrrF, PrrF2 and CrcZ), reported to have role in its pathogenicity was detected in all keratitis strains. Out of 130 known P. aeruginosa sRNAs, SPA0021 and SPA0023 found to expressed in majority of the MDR/XDR P. aeruginosa keratitis strains. Target gene prediction servers identified, outer membrane porin protein OprD was one of the binding target for SPA0023 with high binding affinity. Several studies have been reported the increased imipenem resistance with inactivation and negative regulation of OprD. However, further functional studies with greater number of $P$. aeruginosa keratitis strains are warranted to confirm the negative regulation of OprD by SPA0023.

\section{Competing Interest Statement}

The authors have declared no competing interest.

\section{Funding}

Aravind Medical Research Foundation, Madurai, India.

\section{References}

[1] Stover C, Pham XQT, Erwin A, Mizoguchi SD, Warrener P, Hickey MJ, et al. Complete genome sequence of Pseudomonas aeruginosa PAO1, an opportunistic pathogen. Nature 2000;406:959-964. doi: $10.1038 / 35023079$

[2] Richards MJ, Edwards JR, Culver DH, Gaynes RP. Nosocomial infections in medical intensive care units in the United States. Critical Care Medicine 1999;27:887-892. 
[3] Green M, Apel A, Stapleton F. Risk Factors and Causative Organisms in Microbial Keratitis. Cornea 2008;27:22-27. doi: 10.1097/ICO.0b013e318156caf2

[4] Hooi S. Culture-proven bacterial keratitis in a Malaysian General Hospital. The Medical journal of Malaysia 2006;60:614-623.

[5] Parmar P, Salman A, Kalavathy CM, Kaliamurthy J, Thomas PA, Jesudasan AN. Microbial keratitis at extremes of age. American journal of ophthalmology 2006;142:204. doi: 10.1016/j.ajo.2006.05.018

[6] Sharma N, Sinha R, Singhvi A, Tandon R. Pseudomonas keratitis after laser in situ keratomileusis. Journal of cataract and refractive surgery 2006;32:519-521. doi: 10.1016/j.jcrs.2005.12.061

[7] Mathee K, Narasimhan G, Valdes C, Carter M, Matewish J, Koehrsen M, et al. Dynamics of Pseudomonas aeruginosa genome evolution. Proceedings of the National Academy of Sciences of the United States of America 2008;105:3100-3105. doi: 10.1073/pnas.0711982105

[8] Goodman AL, Lory S. Analysis of regulatory networks in Pseudomonas aeruginosa by genomewide transcriptional profiling. Current Opinion in Microbiology 2004;7:39-44. doi: https://doi.org/10.1016/j.mib.2003.12.009

[9] Kandasamy K, Thirumalmuthu K, Prajna NV, Lalitha P, Mohankumar V, Devarajan B. Comparative genomics of ocular Pseudomonas aeruginosa strains from keratitis patients with different clinical outcomes. Genomics 2020;112:4769-4776.

[10] Subedi D, Vijay AK, Kohli GS, Rice SA, Willcox M. Comparative genomics of clinical strains of Pseudomonas aeruginosa strains isolated from different geographic sites. Scientific Reports 2018;8:15668. doi: 10.1038/s41598-018-34020-7

[11] Khan M, Willcox M, Stapleton F, Summers S, Rice S. Antibiotic Resistance Characteristics of Pseudomonas aeruginosa Isolated from Keratitis in Australia and India. Antibiotics 2020;9:600. doi: 10.3390/antibiotics9090600

[12] Nandagopal M, Jambulingam M, Vetrivel DU, Madhavan H. Unraveling Genomic and Phenotypic nature of Multidrug-Resistant (MDR) Pseudomonas aeruginosa VRFPA04 Isolated From Keratitis Patient. Microbiological Research 2016;193:140-149. doi: 10.1016/j.micres.2016.10.002

[13] Stewart R, Wiehlmann L, Ashelford K, Preston S, Frimmersdorf E, Campbell B, et al. Genetic Characterization Indicates that a Specific Subpopulation of Pseudomonas aeruginosa Is Associated with Keratitis Infections. Journal of clinical microbiology 2011;49:993-1003. doi: 10.1128/jcm.02036-10

[14] Lenz D, Mok K, Lilley B, Kulkarni R, Wingreen N, Bassler B. The Small RNA Chaperone Hfq and Multiple Small RNAs Control Quorum Sensing in Vibrio harveyi and Vibrio cholerae. Cell 2004;118:69-82. doi: 10.1016/j.cell.2004.06.009

[15] Argaman L, Hershberg R, Vogel J, Bejerano G, Wagner EGH, Margalit H, et al. Novel small RNAencoding genes in the intergenic regions of Escherichia coli. Current Biology 2001;11:941-950. doi: https://doi.org/10.1016/S0960-9822(01)00270-6

[16] Heurlier K, Williams F, Heeb S, Dormond C, Pessi G, Singer D, et al. Positive Control of Swarming, Rhamnolipid Synthesis, and Lipase Production by the Posttranscriptional RsmA/RsmZ System in Pseudomonas aeruginosa PAO1. Journal of bacteriology 2004;186:2936-2945. doi: 10.1128/jb.186.10.2936-2945.2004

[17] Law C, Huang C, Pan Q, Lee J, Hao Q, Chan T-F, et al. A small RNA transforming multidrug resistance Pseudomonas aeruginosa to drug-susceptibility. Molecular Therapy - Nucleic Acids 2019;16 doi: 10.1016/j.omtn.2019.02.011

[18] Brantl S. Regulatory mechanisms employed by cis-encoded antisense RNAs. Current Opinion in Microbiology 2007;10:102-109. doi: https://doi.org/10.1016/j.mib.2007.03.012

[19] Valentin-Hansen P, Eriksen M, Udesen C. The bacterial Sm-like protein Hfq: A key player in RNA transactions. Molecular microbiology 2004;51:1525-1533. doi: 10.1111/j.1365-2958.2003.03935.x

[20] Aiba H. Mechanism of RNA silencing by Hfa-binding small RNAs. Current Opinion in Microbiology 2007;10:134-139. doi: https://doi.org/10.1016/j.mib.2007.03.010 
[21] Regnier P, Hajnsdorf E. The role of the RNA chaperone $\mathrm{Hfq}$ in Poly $(\mathrm{A})$ metabolism : methods to determine positions, abundance and lengths of seldom and short oligo (A) tails. 2008

[22] Morita T, Maki K, Yagi M, Aiba H. Chapter 18 Analyses of mRNA Destabilization and Translational Inhibition Mediated by Hfq-Binding Small RNAs. Methods in Enzymology: Academic Press, 2008. p. 359378.

[23] Magiorakos AP, Srinivasan A, Carey RB, Carmeli Y, Falagas ME, Giske CG, et al. Multidrug-resistant, extensively drug-resistant and pandrug-resistant bacteria: an international expert proposal for interim standard definitions for acquired resistance. Clinical Microbiology and Infection 2012;18:268-281. doi: https://doi.org/10.1111/j.1469-0691.2011.03570.x

[24] Treangen TJ, Ondov BD, Koren S, Phillippy AM. The Harvest suite for rapid core-genome alignment and visualization of thousands of intraspecific microbial genomes. Genome Biology 2014;15:524. doi: 10.1186/s13059-014-0524-x

[25] Grenfell-Lee D, Urbach J, Wu G, Liberati N, Feinbaum R, Miyata S, et al. Genomic analysis reveals that Pseudomonas aeruginosa virulence is combinatorial. Genome Biology 2006;7:R90. doi: 10.1186/gb2006-7-10-r90

[26] Roy P, Tetu S, Larouche A, Elbourne L, Tremblay S, Ren Q, et al. Complete Genome Sequence of the Multiresistant Taxonomic Outlier Pseudomonas aeruginosa PA7. PloS one 2010;5:e8842. doi: 10.1371/journal.pone.0008842

[27] Letunic I, Bork P. Interactive Tree Of Life (iTOL) v5: an online tool for phylogenetic tree display and annotation. Nucleic acids research 2021;49:W293-W296. doi: 10.1093/nar/gkab301

[28] Alikhan N-F, Petty N, Ben Zakour N, Beatson S. BLAST Ring Image Generator (BRIG): simple prokaryote genome comparisons. BMC Genomics 2011;12:402. doi: 10.1186/1471-2164-12-402

[29] Kery M, Feldman M, Livny J, Tjaden B. TargetRNA2: Identifying targets of small regulatory RNAs in bacteria. Nucleic acids research 2014;42 doi: 10.1093/nar/gku317

[30] Mann M, Wright PR, Backofen R. IntaRNA 2.0: enhanced and customizable prediction of RNA-RNA interactions. Nucleic acids research 2017;45:W435-W439. doi: 10.1093/nar/gkx279

[31] Dennis G, Sherman BT, Hosack DA, Yang J, Gao W, Lane HC, et al. DAVID: Database for Annotation, Visualization, and Integrated Discovery. Genome Biology 2003;4:R60. doi: 10.1186/gb-2003-4-9-r60

[32] Kanehisa M, Goto S. KEGG: kyoto encyclopedia of genes and genomes. Nucleic acids research 2000;28:27-30. doi: 10.1093/nar/28.1.27

[33] Maertzdorf J, McEwen G, Weiner J, 3rd, Tian S, Lader E, Schriek U, et al. Concise gene signature for point-of-care classification of tuberculosis. EMBO Mol Med 2016;8:86-95. doi: 10.15252/emmm.201505790

[34] Freschi L, Jeukens J, Kukavica-Ibrulj I, Boyle B, Dupont M-J, Laroche J, et al. Clinical utilization of genomics data produced by the international Pseudomonas aeruginosa consortium. Front Microbiol 2015;6 doi: 10.3389/fmicb.2015.01036

[35] Stewart L, Ford A, Sangal V, Jeukens J, Boyle B, Kukavica-lbrulj I, et al. Draft genomes of 12 hostadapted and environmental isolates of Pseudomonas aeruginosa and their positions in the core genome phylogeny. Pathogens and disease 2014;71:20-25. doi: 10.1111/2049-632x.12107

[36] Kos VN, Déraspe M, McLaughlin RE, Whiteaker JD, Roy PH, Alm RA, et al. The Resistome of Pseudomonas aeruginosa in Relationship to Phenotypic Susceptibility. Antimicrobial Agents and Chemotherapy 2015;59:427-436. doi: doi:10.1128/AAC.03954-14

[37] He J, Baldini R, Déziel E, Saucier M, Zhang $Q$, Liberati $N$, et al. The broad host range pathogen Pseudomonas aeruginosa strain PA14 carries two pathogenicity islands harboring plant and animal virulence genes. Proceedings of the National Academy of Sciences of the United States of America 2004;101:2530-2535. doi: 10.1073/pnas.0304622101

[38] Fang Z-L, Zhang L-Y, Huang Y-M, Qing Y, Cao K-Y, Tian G-B, et al. OprD mutations and inactivation in imipenem-resistant Pseudomonas aeruginosa isolates from China. Infection, genetics and evolution : 
journal of molecular epidemiology and evolutionary genetics in infectious diseases 2013;21 doi: 10.1016/j.meegid.2013.10.027

[39] Ochs M, McCusker M, Bains M, Hancock R. Negative Regulation of the Pseudomonas aeruginosa Outer Membrane Porin OprD Selective for Imipenem and Basic Amino Acids. Antimicrobial Agents and Chemotherapy 1999;43:1085-1090. doi: 10.1128/aac.43.5.1085

[40] Kim CH, Kang HY, Kim BR, Jeon H, Lee YC, Lee SH, et al. Mutational inactivation of OprD in carbapenem-resistant Pseudomonas aeruginosa isolates from Korean hospitals. Journal of Microbiology 2016;54:44-49. doi: 10.1007/s12275-016-5562-5

[41] Wolter DJ, Hanson ND, Lister PD. Insertional inactivation of oprD in clinical isolates of Pseudomonas aeruginosa leading to carbapenem resistance. FEMS Microbiology Letters 2004;236:137143. doi: 10.1111/j.1574-6968.2004.tb09639.x 